UNIWERSYTET IM. ADAMA MICKIEWICZA W POZNANIU

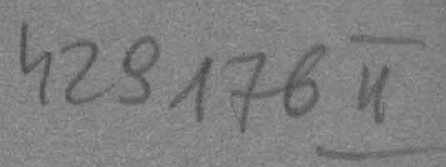

XV
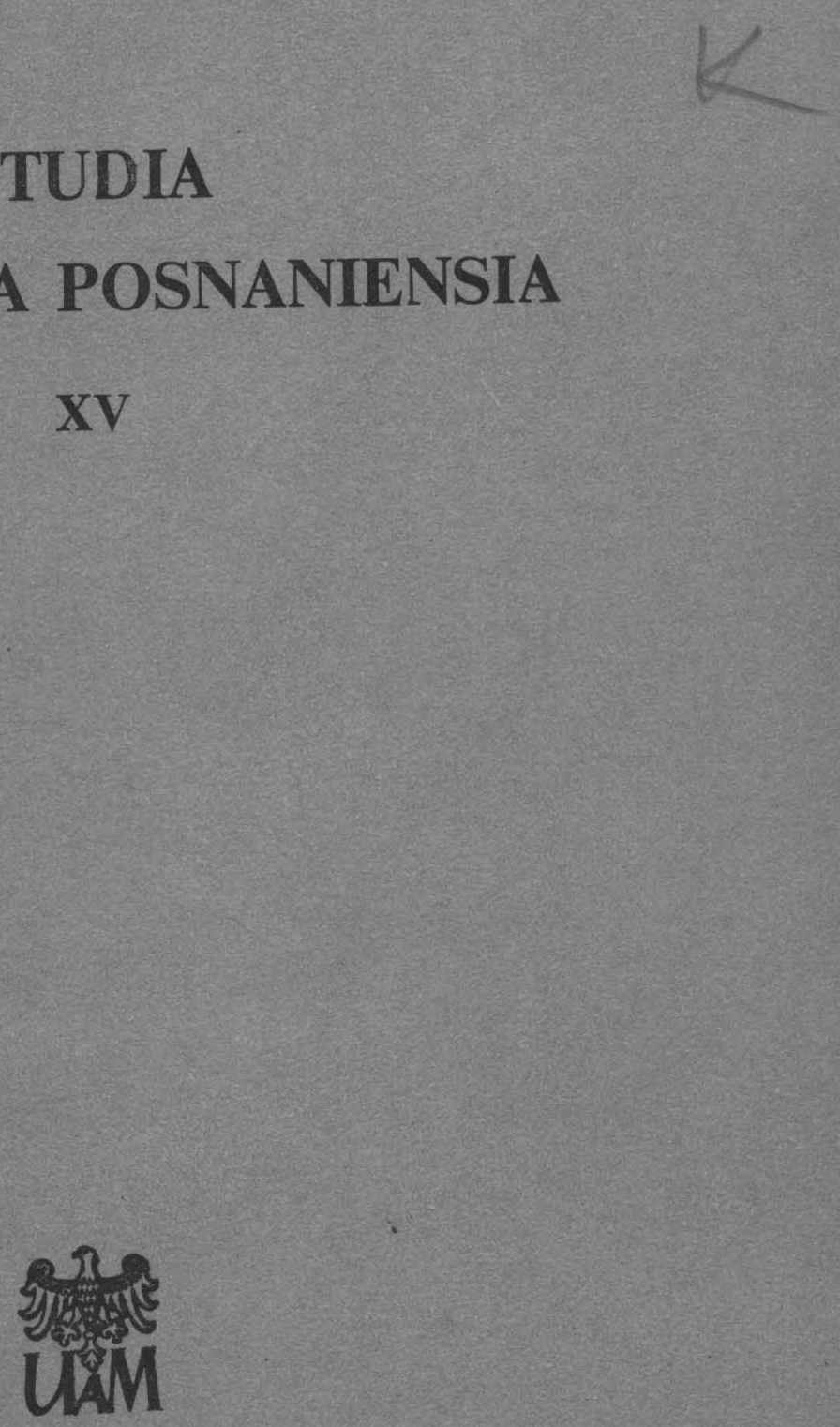

POZNAŃ 1986 

UNIWERSYTET IM. ADAMA MICKIEWICZA W POZNANIU

\section{STUDIA \\ GERMANICA POSNANIENSIA}

\section{XV}

Sprachwissenschaft
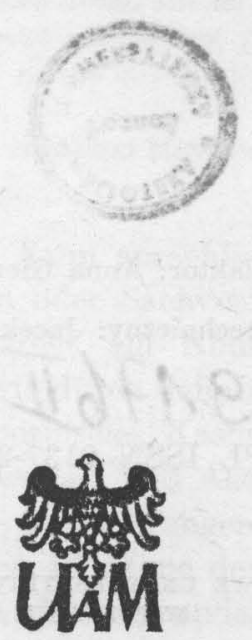

POZNAŃ 1986 


\section{Redaktor naukowy}

ANDRZEJ Z. BZDĘGA

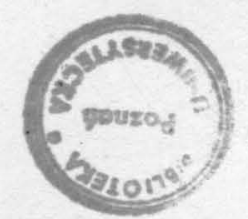

Redaktor: Anna Gierlińska

Redaktor techniczny: Jacek \&rześkowiak

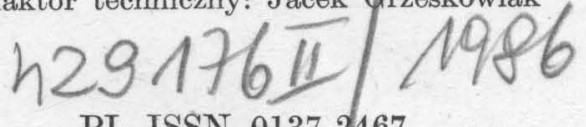

PL ISSN 0137-2467

WYDAWNICTWO NAUKOWE UNTWERSYTETU IM. ADAMA MICKIEWICZA W POZNANIU

Nakład 520+80 egz. Ark. wyd. 12,75. Ark. druk. 10,875. Papier druk. sat. kl. III. $80 \mathrm{~g}$ $70 \times 100$. Oddano do składania w październiku 1985 r. Podpisano do druku w listopadzie 1986 r. Druk ukończono w listopadzie 1986 r. Zam. nr 76/150. Cena zl 130,DRUKARNIA UNIWERSYTETU IM. ADAMA MICKIEWICZA - POZNAÑ. UL. FREDRY 10 
Andrzej Z. Bzdęga (Poznań): Soziativa im Deutschen und Polnischen . . . . . . 3

Józef Darski (Poznań): Syntaktische Exponenten der Lexeme . . . . . . . . 17

Alicja Gaca (Poznań): Deutsch-polnische Äquivalenz aus der Sicht der Textstruk. . . . . . . . . . .

Józef Grabarek (Rzeszów): Zur Auflösung der Nulläquivalenz im nominalen Berøich bei der Translation aus dem Deutschen ins Polnische . . . . . . . . .

Iwona Jankowska (Szczecin): Zur Semantik der Tages- und Epochenbezeichnungen im Deutschen und Polnischen . . . . . . . . . . . . . . . .

Gabriela Koniuszaniec (Poznań): Zur adverbalen Funktion der für-Phrase aus kontrastiver Sicht . . . . . . . . . . . . . . . . . . . . .

Sławomir Mikołajczak (Poznań): Die Konstituentenstruktur der adjektivischen Komposita im Deutschen und Polnischen . . . . . . . . . . . . .

Izabela Prokop (Poznań): Exozentrische Konstruktionen im Deutschen und Polnischen . . . . . . . . . . . .

Izabela Prokop (Poznań): Antworten auf Ergänzungsfragen im Deutschen und Polnischen

\section{BIBLIOGRAPHIE}

Andrzej Kątny (Rzeszów): Bibliographie zur deutsch-polnischen Konfrontation, Teil II . . . . . . . . . . . . . . . . . . . . . .

\section{BERICHTE}

Jan Papiór (Poznań): Institut für Germanische Philologie der Adam-MickiewiczUniversität Poznań ....................

\section{REZENSIONEN}

Eugeniusz Tomiezek, System adresatywny współczesnego języka polskiego i niemieckiego, Wrocław 1983 (Izabela Prokop) . . . . . . . . . . . .

Helgard Lörcher, Gesprächsanalytische Untersuchungen zur Arzt-Patienten-Kommunikation, Tübingen 1983 (Izabela Prokop) . . . . . . . . . . . 
Einführung in die konfrontative Linguistik, Leipzig 1983 (Anne Przybecka) . . . 164 Jan Jeziorski, Substantivische Nominalkomposita des Deutschen und ihre polnischen Entsprechungen, Wrocław 1983 (Andrzej Z. Bzdęga) . . . . . . . . Jindřich Toman, Wortsyntax. Eine Disskusion ausgewählter Probleme deutscher Wortbildung, Tübingen 1983 (Andrzej Z. Bzdęga) . . . . . . . 168

\section{NEKROLOGE}

Maria Wrzeciono 1912 - 1984 (Cecylia Załubska) 171 


\title{
ZUR SEMANTIK DER TAGES- UND EPOCHENBEZEICHNUNGEN IM DEUTSCHEN UND POLNISCHEN
}

\begin{abstract}
Iwona Jankowska, Zur Semantik der Tages- und Epochenbezeichnungen im Deutschen und Polnischen [The analysis of meaning of time expressions of the time of day and epoch], Studia Germanica Posnaniensia, Adam Mickiewicz University Press, Poznań, vol. XV: 1986, pp. 51-65, PL ISSN 0137-2467.

The article refers to $\mathrm{Ph} . \mathrm{D}$. dissertation in which the author investigated adverbials of time in German and Polish. Using a similar method of analysis, namely establishing the relations occurring between the point of time of the event, the reference and the speech act, the author describes the usage of definitions of a day and longer periods (epochs). The author refers at the same time to simple formulas "of formal logic and juxtaposes all the relevant expressions in both languages.
\end{abstract}

Iwona Jankowska, Department of German, University of Szczecin, Poland.

Unter vielen temporalen Ausdrücken, die sich auf die Einheiten der öffentlichen Zeitrechnung beziehen, bilden die Adverbien zur Bezeichnung der Tage und Epochen eine besondere Gruppe. Das Besondere an diesem relativ kleinen und geschlossenen lexikalischen Teilbereich ist die Tatsache, daß die einzelnen Adverbien im Unterschied zu den Kalenderausdrücken eng mit dem Sprechzeitpunkt verbunden sind, und zwar so, daß er für sie die einzige Möglichkeit des Bezugspunktes bildet. Nur für das polnische Adverb nazajutrz 'am nächsten Tag' trifft diese Feststellung nicht zu. Darauf werden wir noch später zu sprechen kommen.

Bevor wir zu den weiteren Überlegungen zur Semantik der einzelnen Glieder dieses lexikalischen Teilbereichs übergehen, wollen wir die Adverbien zur Bezeichnung der Tage und Epochen in einer Tabelle zusammenstellen, die außer den Adverblexemen auch die Informationen über die Verteilung ihrer Interpretationen und die Möglichkeiten der Adjektivbildung enthält:

1 Die zur Untersuchung herangezogenen Adverbien wurden den folgenden Wörterbüchern entnommen: A. Bzdęga, J. Chodera, S. Kubica, Podręczny slownite polsko- 
I. Jankowska

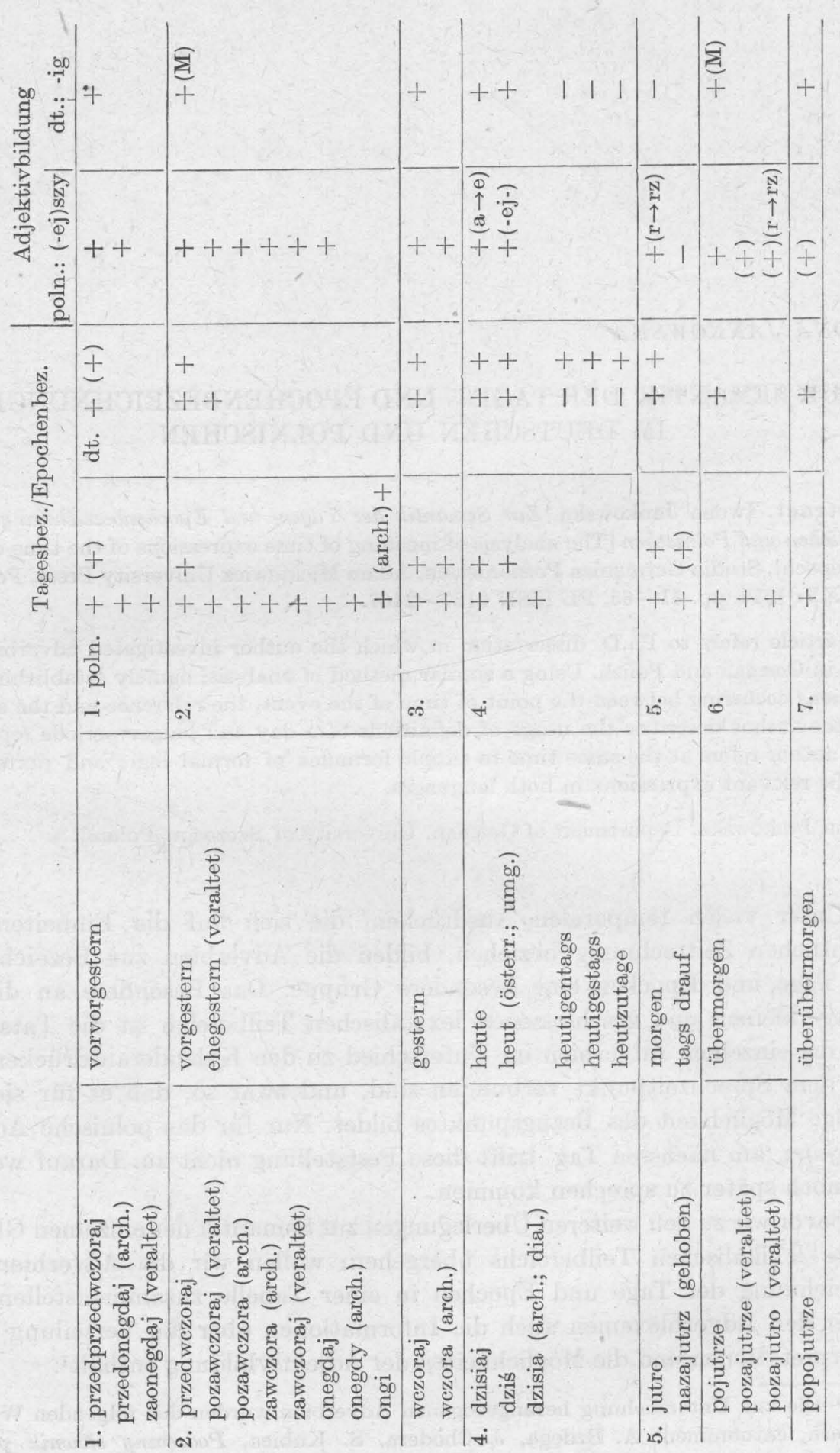


Zunächst wollen wir unsere Aufmerksamkeit den Tagesadverbien zuwenden. Es sind alles Bezeichnungen für Intervalle von gleicher Länge, und zwar von 24 Stunden, die sich untereinander in der Zahl der Intervalle und in der Richtung ihrer Entfernung vom Sprechzeitpunkt unterscheiden. Der Sprechzeitpunkt ist dabei, wie bereits eingangs angedeutet wurde, der einzige mögliche Bezugspunkt für diese Adverbien, was folgendermaßen notiert werden könnte ${ }^{2}$ :

$$
t_{B}=t_{s} \&\left(\sim\left(\left(t_{B} \text { vor } t_{s}\right) \vee\left(t_{B} \text { nach } t_{s}\right)\right)\right)
$$

Der Zeitpunkt des Ereignisses, der zu einem dieser Intervalle gehört, kann somit entweder vor dem Sprechzeitpunkt oder danach liegen. Da aber der Sprechzeitpunkt auch zu einem Zeitintervall, und zwar zu heute/dzisiaj gehört, erweist es sich als notwendig, für die jeweilige Distanz zwischen $t_{\mathrm{s}}$ und $t_{\mathrm{F}}$ maximale und minimale Länge festzustellen. Dabei gilt ständig, daß:

$$
\left(t_{\mathrm{s}} \subset \mathrm{T}_{\mathrm{E}(0-24)_{\mathrm{n}}}\right) \&\left(\mathrm{t}_{\mathrm{E}} \subset \mathrm{T}_{\mathrm{E}(0-24)_{\mathrm{m}}}\right)
$$

wenn: $\mathrm{n}=1$

$$
\mathrm{m} \geqslant 1 \sim(\mathrm{m}>4)
$$

$0=$ Anfang von heute $=0 \mathrm{Uhr}$

Bei heute/dzisiaj lassen sich die maximale und die minimale Distanz zwischen $t_{\mathrm{s}}$ und $t_{\mathrm{E}}$ etwa in folgender Weise ausrechnen:

-niemiecki, Bd. I/II, Ẇarszawa 1980; W. Doroszewski, Stownik języka polskiego (in 11 Bänden) (SJP); W. Doroszewski, Slownik poprawnej polszczyzny, Warszawa 1977; R. Klappenbach, W. Steinitz, Wörterbuch der deutschen Gegenwartssprache, Bd. 1-6, Berlin 1978 (WDG); H. Klien (Hrsg.), Der Große Duden, Wörterbuch und Leitfaden der deutschen Rechtschreibung, Leipzig 1969 (D); E. Mater, Rückläufiges Wörterbuch der deutschen Gegenwartssprache, Leipzig 1965 (M); J. Piprek, J. Ippoldt, Wielli slownik polsko-niemiecki, Bd. 1-2, Warszawa 1971; J. Piprek, J. Ippoldt, Wielki stownik niemiecko-polski, Bd. 1-2, Warszawa 1972 (Piprek, Ippoldt); G. Wahrig, Deutsches Wörterbuch, Gütersloh 1968.

2 In der Untersuchung werden folgende Zeichen und Symbole verwendet:

$t_{\mathrm{s}}=$ der Sprechzeitpunkt (als Spezialfall des Bezugspunktes)

$t_{B}=$ der Bezugspunkt (der Punkt, zu dem der Ereignismoment in die Relation 〈vor〉, $\langle$ nach $\rangle,\langle$ gleich $(=)\rangle$ gesetzt wird)

$\mathrm{t}_{\mathrm{E}}=$ der Ereignismoment

$\mathrm{T}=$ die Zeitspanne, zu der die einzelnen Zeitpunkte gehören

$\&=$ Zeichen der Konjunktion

$\mathrm{V}=$ Zeichen der Disjunktion

$c=$ Zeichen der Inklusion

$\rightarrow=$ Zeichen der Implikation

$\sim=$ Zeichen der Negation

$>=$,größer als"

$\geqslant=$,gleich oder größer als"

m, n=Variablen über die Zahl der Zeiteinheit ,Tag'

*=(vor den Beispielen) bezeichnet die sprachlich nicht akzeptablen Formen 
$\max$ Dist $\left(\mathrm{t}_{\mathrm{s}}, \mathrm{t}_{\mathrm{E}}\right)=24-\min$ Dist $\left(0, \mathrm{t}_{\mathrm{s}}\right)=24-0,000 \ldots 1=23,999 \ldots 9$

$\min$ Dist $\left(t_{s}, t_{E}\right)=24-\max$ Dist $\left(0, t_{s}\right)=24-23,999 \ldots 9=0,000 \ldots 1$

Wenn sich der Sprechakt mit der Sprechzeit deckt, so in Äußerungen mit den Verben vom Typ taufen, ernennen im Präsens, gibt es überhaupt keine zeitliche Distanz zwischen $t_{\mathrm{s}}$ und $t_{\mathrm{E}}$.

Da für heute/dzisiaj die Bedingung $\mathrm{m}=1$ zutrifft, läßt sich die allgemeine Notation des Verhältnisses zwischen $t_{\mathrm{s}}$ und $\mathrm{t}_{\mathrm{E}}$ wie folgt spezifizieren:

$$
\left(\left(\mathrm{t}_{\mathrm{s}} \subset \mathrm{T}_{\mathrm{E}(0-24)}\right) \&\left(\mathrm{t}_{\mathrm{E}} \subset \mathrm{T}_{\mathrm{E}(0-24)}\right)\right) \rightarrow\left(\left(\mathrm{t}_{\mathrm{s}}, \mathrm{t}_{\mathrm{E}}\right) \subset \mathrm{T}_{\mathrm{E}(0-24)}\right)
$$

Zwischen dem Sprechzeitpunkt und dem Ereignismoment kann sowohl die Relation 〈vor〉 als auch die Relation 〈nach〉 eintreten. Damit hängt die Tatsache zusammen, daß heute/dzisiaj mit einem Vergangenheits- aber auch mit einem Zukunftstempus gebraucht werden können ${ }^{3}$ :

(1 a) Ich war heute im Kino.

(1 b) Byłam dzisiaj w kinie.

(2 a) Er wird dich heute anrufen./Er ruft dich heute an.

(2 b) Będzie dzwonil do ciebie dzisiaj.|Dzisiaj do ciebie zadzwoni.

Alle anderen Tagesadverbien lassen nur eine dieser Relationen zu:

$$
\left(t_{B}=t_{s}\right) \&\left\{\begin{array}{l}
\left(t_{E} \text { vor } t_{B}\right) \rightarrow\left(t_{E} \text { vor } t_{s}\right) \\
\left(t_{E} \text { nach } t_{B}\right) \rightarrow\left(t_{E} \text { nach } t_{s}\right)
\end{array}\right\} \&
$$

$\min$ Dist $\left(t_{s}, t_{E}\right) \& \max$ Dist $\left(t_{s}, t_{E}\right)$

Die Notationen für die minimale und maximale Distanz zwischen $t_{\mathrm{s}}$ und $t_{\mathrm{E}}$ können für morgen/jutro und gestern/wczoraj in Anlehnung an die entsprechende Notation von heute/dzisiaj formuliert werden:

morgen/jutro

$\max$ Dist $\left(\mathrm{t}_{\mathrm{s}}, \mathrm{t}_{\mathrm{E}}\right)=\left(24+\min\right.$ Dist $\left.\left(0, \mathrm{t}_{\mathrm{s}}\right)\right)+24=23,999 \ldots 9+24=47,999 \ldots 9$

$\min$ Dist $\left(t_{\mathrm{s}}, \mathbf{t}_{\mathbf{E}}\right)=\left(24-\max\right.$ Dist $\left.\left(0, t_{\mathrm{s}}\right)\right)+\min \operatorname{Dist}\left(0, t_{\mathrm{E}}\right)=$ $=(24-23,999 \ldots 9)+0,000 \ldots 1=0,000 \ldots 2$

${ }^{3} \mathrm{Zu}$ diesem Problem siehe u.a.: D. Wunderlich, Tempus und Zeitreferenz im Deutschen, München 1970; R. Bäuerle, Tempus und Temporaladverb, Linguistische Berichte 50, 1977, S. 51-57; R. Bäuerle, Temporale Deixis, temporale Frage, Tübingen 1979. 
gestern/wczoraj

$\max \operatorname{Dist}\left(\mathrm{t}_{\mathrm{s}}, \mathrm{t}_{\mathrm{E}}\right)=\max$ Dist $\left(0, \mathrm{t}_{\mathrm{s}}\right)+24=23,999 \ldots 9+24=47,999 \ldots 9$

$\min$ Dist $\left(t_{\mathrm{s}}, \mathrm{t}_{\mathrm{E}}\right)=\min$ Dist $\left(0, \mathrm{t}_{\mathrm{s}}\right)+\min$ Dist $\left(0, \mathrm{t}_{\mathrm{E}}\right)=$ $=0,000 \ldots 1+0,000 \ldots 1=0,000 \ldots 2$

Die Notationen zeigen, daß die maximale und minimale Distanz zwischen $t_{\mathrm{s}}$ und $t_{\mathbf{E}}$ für die beiden Tagesbezeichnungen gleich sind. Die semantischen Notationen dieser Adverbien unterscheiden sich nur in einem Punkt, und zwar in der Richtung der temporalen Relation zwischen $t_{s}$ und $t_{\mathbf{E}}$ : bei morgen/jutro erscheint $t_{\mathrm{E}}$ nach $t_{\mathrm{s}}$, bei gestern/wczoraj $t_{\mathrm{E}}$ vor $t_{\mathrm{s}}$.

Was die anderen Tagesbezeichnungen betrifft, so lassen sich ihre Notationen von denen bei morgen/jutro und gestern/wczoraj ableiten, und zwar durch die Addition von je 24 Stunden für jedes vorwärts bzw. rückwärts nächstfolgende Tagesadverb. Theoretisch ist es dabei möglich, diese 24 Stunden unbegrenzt zu addieren, da die Tage in ihrer Zahl auch keiner Begrenzung unterliegen. Diese Möglichkeit gibt es theoretisch auch in der Wortbildung solcher Formen, da die in den semantischen Notationen von morgen/jutro und gestern/wczoraj abgeleiteten Adverbien sprachlich mit Hilfe der Präfixe über-/po- und vor-/ Iprzed- realisiert werden können. Aus Gründen der Verständlichkeit ist es dabei üblich, diese Präfixe höchstens doppelt zu gebrauchen: übermorgen/pojutrze, überübermorgen/popojutrze; vorgestern/przedwczoraj, vorvorgestern/przedprzedwczoraj. Die bei den veralteten Adverbien verwendeten Präfixe ehe-|poza-, $z a$ - treten allerdings nie verdoppelt auf. So finden wir z.B. die Formen: pozajutrze, pozajutro 'übermorgen' oder zawczoraj, pozawczorajlehegestern und nicht *pozapozajutrze 'überübermorgen', *zazawczoraj 'vorvorgestern' oder * eheehegestern.

Bei der Distanz zwischen $t_{\mathrm{s}}$ und $t_{\mathrm{E}}$, die größer als drei Tage ist, werden Adverbiale mit entsprechenden Numeralen verwendet, z.B. vor vier Tagen/ Iprzed czterema dniami, cztery dni temu; in vier Tagen/za catery dni. Die Formen *vorvorvorgestern/*przedprzedprzedwczoraj oder *überüberübermorgen/*popopojutrze sind nicht mehr akzeptabel. Die üblichen Tagesadverbien können auch in solche adverbialen Konstruktionen aufgelöst werden. Eine Ausnahme bilden die Grundtagesadverbien, deren maximale Distanz zwischen $\mathrm{t}_{\mathrm{s}}$ und $\mathrm{t}_{\mathrm{E}} 47,999 \ldots 9$ und die minimale Distanz $0,000 \ldots 2$ Stunden beträgt, d.h. morgen/jutro und gestern/wczoraj. Die entsprechende Konstruktion mit dem Numerale ein/jeden ist weder im Deutschen noch im Polnischen gebräuchlich:

(3 a) Das Symposium begann vorgestern/vor zwei Tagen.

(3 b) Sympozjum rozpoczęło się przedwczoraj।przed dwoma dniami/dwa dni temu. 
(4 a) Das Symposium beginnt übermorgen/in zwei Tagen.

(4 b) Sympozjum rozpocznie się pojutrze/za dwa dni.

(5 a) Helga ist gestern/*vor einem Tag nach Berlin gefahren.

(5 b) Helga pojechała wczoraj|*przed jednym dniem/*jeden dzień temu do Berlina.

(6 a) Sie kommt morgen/*in einem Tag nach Leipzig zurück.

(6 b) Jutro/*za jeden dzień wraca do Lipska.

Sowohl das Deutsche als auch das Polnische besitzen die Bezeichnungen für die gleiche Zahl der Tage, und zwar für insgesamt je sieben Zeiteinheiten dieser Art. Aus der Tabelle auf S. 52 geht dabei deutlich hervor, daß fast jeder von diesen Zeiteinheiten mehrere Bezeichnungen zugeordnet werden. Dies trifft vor allem für das Polnische zu. Die meisten Bezeichnungen werden aber in der Gegenwartssprache nicht mehr verwendet. Für die deutsche Sprache geben die Wörterbücher nur ein veraltetes Tagesadverb an, und zwar ehegestern. Die Form, heut wird im österreichischen Sprachraum als normal- und sonst als úmgangssprachlich empfunden. Insgesamt werden den sieben Zeiteinheiten zweiundzwanzig polnische und neun deutsche Tagesadverbien zugeordnet.

Daß nicht jede Sprache Bezeichnungen für die gleiche Zahl der Zeiteinheit ,Tag' besitzt, läßt sich u.a. am Beispiel des Schwedischen zeigen. Es hat drei Grundtagesbezeichnungen: idag 'heute', igår 'gestern', i morgon 4 'morgen'. Zwei weitere werden ähnlich wie im Deutschen und Polnischen gebildet: przedwczoraj - vorgestern - i förrgår; pojutrze - übermorgen - i övermorgon.

Wenn aber das Polnische und Deutsche die Wiederholung der ersten Konstituente zulassen, ist es im Schwedischen nicht möglich: przedprzedwczoraj - vorvorgestern - *i förrförrgår; popojutrze - überübermorgen - *i överövermorgon. Die Tageseinheit vorvorgestern wird im Schwedischen mit Hilfe einer umschreibenden Konstruktion bezeichnet, in der der Bezug auf die Tageseinheit vorgestern ausgenutzt wird: dagen före $i$ förrgår 'am Tag vor vorgestern'. Nach einem ähnlichen Schema wird im Englischen die Bezeichnung für die Tageseinheit vorgestern gebildet: a day bevor yesterday 'am Tag vor gestern'. Eine ähnliche Konstruktion besitzt das Englische auch für die Bezeichnung der Tageseinheit übermorgen, die, wie vorgestern auch um zwei Einheiten von heute entfernt ist. Der Unterschied zwischen vorgestern und

4 Die bei den schwedischen Tagesadverbien gebrauchte Präposition $i$ wird bei den Grundtagesbezeichnungen idag und igair oft zusammengeschrieben, obwohl auch die Getrenntschreibung möglich ist, die für die übrigen Tagesadverbien gilt. Dazu vgl.: Svenska akademiens ,Ordlista över svenska språket', Stockholm 1973 oder: E. Wessén, Vara ord, deras uttal och ursprung, Stockholm 1973. 
übermorgen liegt nur in der Richtung der Entfernung dieser Tageseinheiten von der den Sprechzeitpunkt einschließenden Tageseinheit heute. Dies wird im Englischen durch die entsprechende Präposition ausgedrückt. Dem deutschen Adverb übermorgen entspricht also im Englischen die Konstruktion mit der Präposition after: a day after tomorrow. Wenn im Schwedischen für die Tageseinheit vorvorgestern die Präpositionalphrase mit Bezug auf eine andere Tageseinheit, und zwar auf vorgestern, gebildet wird, so ist dieses Schema für die Bezeichnung der Tageseinheit überübermorgen nicht mehr anwendbar. Möglich ist hier nur die präpositionale Konstruktion mit dem Numerale tre 'drei', das die Zahl der Tageseinheiten zum Ausdruck bringt, um die überübermorgen von heute entfernt ist: überübermorgen $=*$ dagen om övermorgon/om tre dagar 'in drei Tagen'. Es zeigt sich also, daß das Englische und Schwedische über weniger Tagesadverbien verfügen als dies im Deutschen und Polnischen der Fall ist: Im Englischen gibt es Tagesadverbien nur für drei, im Schwedischen für fünf aufeinanderfolgende Zeiteinheiten 'Tag'. Es gibt aber auch Sprachen, die Bezeichnungen für mehr als sieben Tage aufweisen. Ch. J. Fillmore nennt hier z.B. das Vietnamesische mit acht solchen Bezeichnungen (davon drei für die Zukunft und vier für die Vergangenheit) oder den Dialekt Chinantec von San Juan Lealao mit insgesamt neun solchen Bezeichnungen, von denen vier die Zukunft und vier die Vergangenheit betreffen. Im gleichen Artikel weist Fillmore auch auf eine andere Möglichkeit für die Bezeichnung der einzelnen Tage hin: ,In manchen Sprachen werden die Tage, die den heutigen Tag umgeben nach ihrer Entfernung von heute benannt, jedoch unabhängig von Zukunft oder Vergangenheit. Hindi z.B. hat Wörter für drei Tage Entfernung: kal bedeutet 'heute'; parsõ bedeutet entweder 'gestern' oder 'morgen'; tarsõ bedeutet 'zwei Tage von heute entfernt' und narsõ bedeutet 'drei Tage von heute entfernt', und zwar in beide Richtungen" 5.

Nach diesem Exkurs über die Tagesbezeichnungen in verschiedênen Sprachen wollen wir nun zu den Tagesadverbien des Polnischen und Deutschen zurückkehren. Es wurde bereits erwähnt, daß für alle Tagesadverbien bis auf poln. nazajutrz die Bedingung $\mathrm{t}_{\mathrm{B}}=\mathrm{t}_{\mathrm{s}}$ gilt. Bei diesem Adverb ist gerade der Sprechzeitpunkt als Bezugspunkt ausgeschlossen. Möglich sind dagegen: $t_{B}$ vor $t_{s}$ und $t_{B}$ nach $t_{s}$, die wiederum für die anderen Tagesadverbien nicht zutreffen. Trotzdem wird nazajutrz, abgesehen von seiner etwas gehobenen Stilfärbung, in den meisten Wörterbüchern, sowohl in den polnischen als auch in den zweisprachigen polnisch-deutschen, mit den Adverbien jutro und morgen gleichgesetzt, mit denen es nur in der Relation $t_{\mathrm{E}}$ nach $\mathrm{t}_{\mathrm{B}}$ übereinstimmt. Daß nazajutrz und jutro/morgen nicht als Äquivalente fungieren können, zeigen

5 Ch. Fillmore, Ansätze zu einer Theorie der Deixis, in: F. Kiefer (Hrsg.), Semantite und generative Grammatik I, Frankfurt a.M. 1972, S. 161. 
die folgenden Sätze:

(7 b) Helga skończy jutro 20 lat.

(7 a) Helga wird morgen 20 Jahre alt.

$\left(t_{B}=t_{s}\right)$

(8 b) *Helga skończy nazajutrz 20 lat.

$\left(t_{B}=t_{s}\right)$

(9 b) Nazajutrz Helga skońezyla 20 lat.

$\left(t_{B}=t_{s}\right)$

$\left(t_{B}\right.$ vor $\left.t_{B}\right)$

(10 b) Nazajutrz/*jutro po powrocie Piotra z Berlina $\left(t_{B}\right.$ vor $\left.t_{s}\right)$ Helga skońezyła 20 lat.

(11 b) Nazajutrz/*jutro po powrocie Piotra z Berlina Helga skończy 20 lat.

$\left(t_{B}\right.$ nach $\left.t_{s}\right)$

Aus den angeführten Sätzen geht deutlich hervor, daß nazajutrz sowohl mit dem Zukunfts- als auch mit dem Vergangenheitstempus kombiniert werden kann, jutro dagegen nur in Sätzen mit dem Zukunftstempus möglich ist, und zwar nur dann, wenn die Bedingung $t_{B}=t_{s}$ erfüllt wird. Bei $\sim\left(t_{B}=t_{B}\right)$ läßt sich nur nazajutrz einsetzen. Die Sätze $(8 \mathrm{~b})-(11 \mathrm{~b})$ zeigen auch, daß bei nazajutrz der Bezugspunkt sprachlich realisiert werden muß, besonders in den Äußerungen mit dem Zukunftstempus, die sonst nicht akzeptiert werden können. Der Bezugspunkt für nazajutrz kann mit Hilfe einer PP, so in (10 b) und $(11 \mathrm{~b})$, eines temporalen Nebensatzes oder des weiteren sprachlichen Kontextes ausgedrückt werden. Möglich sind auch entsprechende Adverbien:

(12 b) W przyszłym tygodniu mój brat ma imieniny.

W piątek rano przyjeżdżają pierwsi goście.

Wieczorem idziemy do opery, a nazajutrz do teatru.

Aus (12 b) geht hervor, daß der Bezugspunkt für nazajutrz zum Intervall 'der nächste Freitag' gehört und nicht zum Intervall 'heute', das den Sprechzeitpunkt enthält. Deshalb können die Tagesadverbien jutro und morgen in einem solchen Kontext nicht gebraucht werden:

(12 b') W przyszłym tygodniu mój brat ma imieniny.

W piątek rano przyjeżdżają pierwsi goście.

Wieczorem idziemy do opery a *jutro do teatru.

An Stelle von morgen kann in (12 a) die kontextgebundene Verbindung tags drauf erscheinen. Möglich ist auch die Präpositionalphrase am nächsten Tag (Abend), die im Polnischen durch die Genetivkonstruktion nastepnego dnia wiedergegeben werden kann:

(12 a) Nächste Woche hat mein Bruder Namenstag. Am Freitagmorgen kommen die ersten Gäste. Abends gehen wir in die Oper und tags drauflam nächsten Tag/*morgen ins Theater. 
Ebenso wenig wie die PP am nächsten Tag oder die Verbindung tags drauf der poln. Tagesbezeichnung jutro entsprechen, können auch die dt. Präpositionalphrase am übernächsten Tag und das polnische Adverb pojutrze gleichgesetzt werden. Trotzdem wird die PP am übernächsten Tag in Piprek, Ippoldt gerade mit Hilfe des polnischen Adverbs pojutrze übersetzt. Daß es nicht richtig ist, zeigt der folgende Satz:

(13 a) Vorigen Freitag hat er mir versprochen, dieses Buch gleich am Sonnabend zurückzugeben. Ich habe es aber erst am übernächsten Tag|*übermorgen von seiner Schwester bekommen.

(13 b) W ubiegły piątek obiecał mi, że odda tę książkę zaraz w sobotę. Dostałam ją jednak dopiero *pojutrze od jego siostry.

Beim Einsetzen der deutschen Verbindung tags drauf für das polnische Adverb nazajutrz muß beachtet werden, daß der kontextuelle Bezugspunkt in tags drauf bereits mit darauf angedeutet wird, bei nazajutrz muß aber erst genannt werden. Deshalb ist tags drauf in der Wiedergabe des polnischen Satzes $(10 \mathrm{~b})$ nicht möglich:

(10 a) *Tags drauf nach Peters Rückkehr aus Berlin ist Helga 20 Jahre alt geworden.

(10 a) könnte nur mit einem Komma akzeptiert werden, wodurch die Verbindung tags drauf und die PP nach Peters Rückkehr aus Berlin syntaktisch nicht mehr voneinander abhängig wären:

(10 a') Tags drauf, (und zwar:) nach Peters Rückkehr aus Berlin, ist Helga 20 Jahre alt geworden.

Es wurde bereits angedeutet, daß die Bedingungen:

$$
\left(t_{E} \text { nach } t_{B}\right) \&\left(t_{B}=t_{B}\right) \& \sim\left(t_{B} \text { vor } t_{s}\right) \& \sim\left(t_{B} \text { nach } t_{s}\right)
$$

die Kombination des Adverbs morgen mit dem Vergangenheitstempus ausschließen. Diese Behauptung steht keineswegs zu folgenden Sätzen in Widerspruch:

(14 a) Falls jemand nach mir fragt: Ich bin morgen verreist.

$$
\text { (nach Gelhaus/Latzel, S. 312) }
$$

(15 a) Ich lag auf der Couch und dachte nach: Morgen um diese Zeit hatte sie schon die Stadt verlassen. (ebenda, S. 319) .

(16 a) Ich befand mich in einem fensterlosen, luftdichten Raum und lag auf der Pritsche. Morgen konnte ich schon nicht mehr atmen ${ }^{6}$. (ebenda, S. $304 \mathrm{f}$.)

- Gelhaus/Latzel vergleichen in diesem Zusammenhang die Austauschbarkeit von morgen und am nächsten Tag und weisen auf den Bedeutungsunterschied zwischen den 
(17 a) Er war froh, daß Zimmering morgen zurückkam.

(A. Seghers, Die Toten bleiben
jung, S. 458)

(18 a) Was war morgen im Fernsehprogramm?

In keinem dieser Sätze nämlich haben die Vergangenheitstempora auch einen Vergangenheitsbezug zu morgen. In (14 a) ist es ein Perfekt mit Zukunftsbezug (als Ersatz für Futur II); in (15 a) und (16 a) wird die Gewißheit ausgedrückt, daß der Sachverhalt in der Zukunft eintritt (16 a) bzw. auch abgeschlossen wird (15 a). Dieser abweichende Gebrauch der Vergangenheitstempora ist, oft mit solchen Störungen der temporalen Linearität wie: Erinnerungen, Rückblenden, Träumen und dgl. verbunden ${ }^{7}$. Auch in (17 a) weist das Präteritum keinen Vergangenheitsbezug auf. Das Tempus des Nebensatzes wird lediglich dem Tempus des Hauptsatzes rein formal angeglichen. Den Nebensatz von (17 a) könnte man genauso gut im Präsens schreiben, ohne einen Bedeutungsunterschied zu bewirken, wobei es auch ein Präsens mit Zukunftsbezug wäre. Dem Satz (18 a) dagegen liegen mehrere Sätze zugrunde, aus denen erst dás Präteritum abgeleitet werden kann, z.B.:

(20 a) - Weißt du, was morgen im Fernsehen ist?

- Ja, sie wollen morgen wieder den Film mit Marlene Dietrich zeigen.

- Entschuldige, ich habe es nicht gehört. Was sagtest du? Was war morgen im Fernsehen?

Das Präteritum yon (18 a) hat zwar die Vergangenheitsbedeutung, im Grunde genommen gehört es aber einem ganz anderen Satz an, in dem morgen nicht gebraucht werden könnte:

(21 a) - *Was sagtest du morgen? Was war im Fernsehen?

Wollen wir die Sachverhalte von (14 a) bis (18 a) im Polnischen wiedergeben, so zeigt sich, daß nur in $(18 \mathrm{~b})$ ein Vergangenheitstempus möglich ist, und

Sätzen (16 a) und (19 a) hin:

(19 a) Ich befand mich in einem fensterlosen, luftdichten Raum. Am nächsten Tag konnte ich kaum noch atmen.

In (16 a) wird , ,die Unmöglichkeit vom Standpunkt des in der Zelle Liegenden aus als gedachte Gewißheit (gedachte Notwendigkeit)" ausgedrückt (S. 304). In (19 a) dagegen wird ,,vom Standpunkt des Erzählers eine Unmöglichkeit als Faktum festgestellt" (ebenda).

7 Vgl. dazu die Arbeiten: K. Hamburger, Die Logik der Dichtung und Noch einmal: Vom Erzählen, in: Euphorion 59/1965; S. Latzel, Zur temporalen Bezugspunktsetzung, in: H. Gelhaus, S. Latzel, Studien zum Tempusgebrauch im Deutschen, Tübingen 1974; F. Stanzel, Episches Präteritum, erlebte Rede, historisches Präsens, Deutsche Vierteljahresschrift für Literaturwissenschaft und Geistesgeschichte, $33 \mathrm{Jg}$. , 1959, S. 1-12. 
zwar aus denselben Gründen wie das Präteritum in (18 a):

(14 b) Jeśli ktoś o mnie zapyta: jutro mnie nie ma, wyjechałem (=powiedz jutro, że wyjechalem).|*Jutro wyjechałem.

(15 b) Leżałem na kanapie i rozmyślałem: Jutro o tej porze nie będzie jej już w mieście/do jutra o tej porze zdąży już opuścić to miasto/ | Jutro o tej porze opuściła to miasto.

(16 b) Znajdowałem się w pomieszezeniu bez okien i dopływu powietrza i leżałem na pryczy.

Jutro nie będę już miał czym oddychać.|*Jutro nie miałem już czym oddychać.

(17 b) *Cieszył się, że Zimmering wrócił jutro./Cieszyl się, że Zimmering ma wrócić jutro.

(18 b) Co to było jutro w telewizji?

(20 b) - Czy wiesz, co jest jutro w telewizji?

- Owszem, jutro mają znowu pokazać ten film z Marleną Dietrich.

- Przepraszam, nie dosłyszałem. Co mówiłaś? Co to bylo jutro w telewizji?

Die Sätze $(14 \mathrm{~b})$ bis (18 b) zeigen, daß die Bedingungen des polnischen Adverbs jutro strenger bei der Kombination mit den Tempora eingehalten werden, als dies im Deutschen der Fall ist. Daß die Sätze (14 a) bis (18 a) tatsächlich möglich sind, besagt jedoch noch nicht, daß sie in dieser Form von den Muttersprachlern als völlig normal akzeptiert werden. Im Gegenteil: Sie werden als äußerst ungewöhnlich empfunden. Eine Ausnahme bildet nur der Satz (18 a), der aber über (20 a) erklärt werden konnte und dem ein ähnlicher Satz im Polnischen entspricht.

Die Tabelle auf S. 52 zeigt, daß einige Tagesadverbien nicht nur die Zeiteinheit ,Tag' betreffen, so dern sich auch ganz allgemein auf die Vergangenheit, Gegenwart oder Zukunft beziehen können und somit über das Intervall von 24 Stunden hinausgehen. Zu diesen Adverbien gehören in erster Linie: heute/ |dzisiaj, dziś; morgen/jutro und gestern/wczoraj. Die Tatsache, daß gerade die Grundtagesbezeichnungen auch als Epochenbezeichnungen fungieren, läßt sich wohl dadurch erklären, daß die Begriffe ,Zukunft' und ,Vergangenheit' nur einerseits, und zwar durch die Gegenwart, begrenzt sind. Sie weisen keinen anderen Grenzpunkt auf, dem der nächste Zeitabschnitt folgen könnte. Wenn also die Tagesadverbien vorgestern/przedwczoraj und übermorgén/pojutrze u.U. auch in der übertragenen Bedeutung als Epochenbezeichnungen verwendet werden, so weisen sie nicht auf den Zeitabschnitt, der nach vs. vor der Zukunft vs. Vergangenheit kommen müßte, sondern auf die Phasen innerhalb dieser 
Zeitabschnitte hin. Diese Adverbien werden dabei als Epochenbezeichnungen nur dann gebraucht, wenn eine sehr weit von der Gegenwart entfernte Phase der Vergangenheit bzw. Zukunft gemeint wird. Meist treten sie dann als adverbiale Attribute auf:

(21 a) Er hat Meinungen von vorgestern.

(21 b) On reprezentuje poglądy z przedwczoraj.

Diese Adverbien weisen dabei noch eine zusätzliche Bedeutung auf: Meinungen von vorgestern sind nicht nur einst gültige, sondern auch überholte und von dem derzeitigen Standpunkt aus von dem Sprecher negativ eingeschätzte Meinungen. An Stelle von vorgestern könnte man in solchen Kontexten auch die Verbindung Anno dazumal einsetzen, die ebenfalls diese negative und etwas pejorativ gefärbte Wertung enthält und der im Polnischen die Bezeichnung z czasów króla Ćwieczka 'aus den Zeiten des legendären Königs Ćwieczek' entspricht. Viele polnische Sprecher würden in solchen Äußerungen wie (21 b) eher diese Bezeichnung als przedwczoraj akzeptieren. Dies hängt damit zusammen, daß im Polnischen die Temporaladverbien äußerst selten als adverbiale Attribute gebraucht werden. Im Deutschen dagegen sind solche Konstruktionen durchaus geläufig. So gilt z.B. die Abkürzung MMM für die Bezeichnung: Die Messe der Meister von Morgen. Jedoch nicht nur vorgestern und przedwczoraj weisen diese wertende Zusatzbedeutung auf. Ein Mensch von heute ist nicht nur ein Mensch, der in der Gegenwart schlechthin lebt, sondern vor allem ,ein moderner Mensch, ein Mensch, der in die Gegenwart paßt" (WDG). Im Gegensatz zu vorgestern und gestern enthält heute meist positive Wertung, es sei denn, es wird in einer Situation verwendet, deren negative Wertung den positiven Inhalt von heute ausschließt, z.B. in der folgenden, oft durch einen Seufzer begleiteten Äußerung:

(22 a) Ach, diese Jugend von heute...

Wenn die Bezeichnung heute meist mit den positiven Vorstellungen verbunden ist, so wird das Attribut von morgen schon mit dom Begriff ,fortschrittlich' gleichgesetzt, so z.B. in (23 a):

(23 a) Er ist ein Mensch von morgen.

Wenn das Adverb vorgestern auch als Epochenbezeichnung fungieren kann, so ist das bei übermorgen kaum möglich. Dies hängt wohl damit zusammen, daß die Vergangenheit als etwas Überlebtes eher in Phasen unterteilt werden kann als die Zukunft, also die Zeit, die erst vor uns liegt und mit keinen Erinnerungen und Erfahrungen verbo rden ist.

Ähnlich wie die deutschen Epochenbezeichnungen weisen auch die polnischen Adverbien dziś, dzisiaj 'heute' und jutro 'morgen' die wertende, und zwar positive Zusatzbedeutung auf. In den Wiedergaben der deutschen ad- 
verbialen Attribute wie in (21 a), (22 a) und (23 a) werden aber im Polnischen keine Adverbien sondern entsprechende adjektivische Ableitungen auf - $(j) s z y$ verwendet:

(22 b) Ach, ta dzisiejsza młodzież...!/ *Ach, ta młodzież $\mathrm{z}$ dzisiaj...!

Die Ableitungen von wczoraj und jutro, sowie auch von przedwczoraj lassen sich allerdings in solchen Kontexten nicht gebrauchen:

(21 b’) On reprezentuje *przedwczorajsze/przestarzałe/średniowieczne poglądy.

Für die Bezeichnung des Überholten wird oft die Verneinung von dzisiejszy, und zwar: niedzisiejszy, verwendet:

$\left(21 \mathrm{~b}^{\prime \prime}\right)$ On reprezentuje niedzisiejsze poglądy.

Sie ist aber stets mit der Vergangenheit verbunden und würde in (23 b) die Bedeutung des Satzes ändern. In (23 b) ist dagegen die Genetivkonstruktion möglich, in der aber nicht das Adverb jutro, sondern das gleichlautende Substantiv vorliegt:

(23 b) On jest człowiekiem jutra/przyszlości. $\neq$ On jest niedzisiejszym człowiekiem.

Auch in der polnischen Wiedergabe der Bezeichnung: Die Messe der Meister von Morgen wird diese Genitivkonstruktion gebraucht: Targi Mistrzów Jutra.

In allen bisherigen Beispielssätzen hatten die Epochenbezeichnungen zusätzliche negative bzw. positive Färbung. Daß es nicht immer so sein muß, zeigt. (24 a), wo die Adverbien gestern, heute und morgen einfach als drei zeitliche Ebenen gegenübergestellt werden:

(24 a) Wie gedacht,

Vor geliebt, jetzt ausgelacht.

Gestern in den Schoß gerissen,

Heute von der Brust geschmissen,

Morgen in die Gruft gebracht.

Wie gedacht,

Vor geliebt, jetzt ausgelacht.

(Günther, S. 29)

In dieser neutralen Interpretation kann auch poln. Adverb nazajutrz gebraucht werden:

(25 b) Mało kto się zastanawia, co będzie nazajutrz.

(25 a) Wenige Leute überlegen, was die Zukunft mit sich bringt. 
Als Epochenbezeichnungen werden außer den bisher besprochenen Adverbien auch die deutschen Zusammenrückungen heutzutage, heutigentags und heutigestages verwendet. Sie beziehen sich auf die Gegenwart, können aber als Tagesadverbien nicht auftreten:

(26 a) Heutzutage will doch keiner mehr arbeiten, jeder will bloß Geld verdienen.

\section{WDG (M. Walser)}

(27 a) *Heutzutage/heute ist er zum erstenmal in die Schule gegangen. Im Polnischen können diese drei Adverbien mit Hilfe des Adverbs dziś/dzisiaj oder auch des Adverbials $w$ dobie dzisiejszej wiedergegeben werden, wobei das Adverbial nur als Epochenbezeichnung gelten kann:

(26 b) Dziś/dzisiaj/w dobie dzisiejszej nikt już nie chce pracować, każdy chce jedynie zarabiać pieniądze.

(27 b) Dziś/dzisiaj|*w dobie dzisiejszej poszedł po raz pierwszy do szkoły.

\section{LITERATUR}

Abraham W./Binnick R. J. (Hrsg.), Generative Semantik, Frankfurt a.M. 1972.

Bal J., Formacje przystówlowe z sufilesalnym j $i$ k typu: dzisiaj, wezoraj, dzisiak, tamok $w$ historii $i$ dialektach języka polskiego, Warszawa 1974.

Bartsch R., Adverbialsemantik, Frankfurt a.M. 1972.

Bäuerle R., Tempus und Temporaladverb, Linguistische Berichte 50, 1977, S. 51-57. -, Temporale Deixis, temporale Frage, Tübingen 1979.

Cyran W., Przystówki polskie. Budowa stowotwórcza, Łódź 1967.

Fillmore Ch., Ansätze zu einer Theorie der Deixis, in: F. Kiefer, Semantik und generative Grammatik I, Frankfurt a.M. 1972, S. 147-174.

Gelhaus H./Latzel S., Studien zum Tempusgebrauch im Deutschen, Tübingen 1974.

Grzegorczykowa R., Typy semantyczne przystówków temporalnych w języku polskim, in: Telest $i$ Jezyk. Problemy semantyczne, Wrocław 1974.

-, Funkcje semantyczne $i$ sktadniowe polskich przyslówków, Warszawa 1975.

-, Zarys stowotwórstwa polskiego. Stowotwórstwo opisowe, Warszawa 1979.

Hamburger K., Die Logik der Dichtung, Euphorion 59/1965.

-, Noch einmal: Vom Erzählen, in: Euphorion 59/1965, S. 46-71.

Jankowska I., Überlegungen zur Semantik der deutschen Temporaladverbien, Studia Germanica Posnaniensia VII/1979.

Jurkowski M., Przyimek $i$ wyrażenia przyimkowe, in: $Z$ polskich studiów slawistycznych, Seria 4, Warszawa 1972, S. 21-29.

Kaufmann G., Zur Temporalangabe im Deutschen, in: Deutschunterricht für Ausländer, 1965 , S. $136-151$.

Kiefer F. (Hrsg.), Semantik und generative Grammatik, Frankfurt a.M. 1972.

Lakoff G., Pronominatisierung, Negation und die Analyse von Adverbien, in: Abraham/ /Binnick, Generative Semantik, S. 116-141.

Latzel S., Zum Gebrauch der deutschen Vergangenheitstempora, in: H. Gelhaus/S. Latzel, Studien zum Tempusgebrauch im Deutschen, Tübingen 1974, S. 169-277. 
Latzel S., Zur temporalen Bezugspunktsetzung, in: Gelhaus/Latzel, Studien zum Tempusgebrauch im Deutschen, S. 277-323.

Stanzel F., Episches Präteritum, erlebte Rede, historisches Präsens, Deutsche Vierteljahresschrift für Literaturwissenschaft und Geistesgeschichte, 33. Jg., 1959, S. 1-12. Steinitz R., Adverbial-Syntax, Berlin 1969.

Steube A., Über die Beziehung der Zeitadverbien zum Satz und ihre Selektionsbeschränkungen zu den relevanten Elementen im Satz, in: Arbeitsstelle Strukturelle Grammatik, Bericht Nr. 3, Januar 1969, S. 1-23.

Steube A., Temporale Bedeutung im Deutschen, Berlin 1980.

Wunderlich D., Über Zeitreferenz und Tempus, Linguistische Berichte 6, S. 1-20.

Wunderlich D., Tempus und Zeitreferenz im Deutschen, München 1970.

Wunderlich D., Pragmatik, Sprechsituation, Deixis, in: Abraham/Binnick, Generative Semantik, S. 285-313. 
